\title{
MARJOLAINE UNTER ECKER, Questions identitaires dans les récits afropéens de Léonora Miano
}

\section{Carminella Biondi}

\section{(2) OpenEdition}

\section{Journals}

\section{Édition électronique}

URL : https://journals.openedition.org/studifrancesi/12251

DOI : 10.4000/studifrancesi. 12251

ISSN : 2427-5856

\section{Éditeur}

Rosenberg \& Sellier

\section{Édition imprimée}

Date de publication : 1 avril 2018

Pagination : 177-178

ISSN : 0039-2944

\section{Référence électronique}

Carminella Biondi, « MARJOLAINE UnTER ECKER, Questions identitaires dans les récits afropéens de Léonora Miano », Studi Francesi [En ligne], 184 (LXII | I) | 2018, mis en ligne le 04 juillet 2018, consulté le 17 novembre 2021. URL : http://journals.openedition.org/studifrancesi/12251 ; DOI : https://doi.org/ 10.4000/studifrancesi. 12251

Ce document a été généré automatiquement le 17 novembre 2021.

\section{(c) (i) (9) $\Theta$}

Studi Francesi è distribuita con Licenza Creative Commons Attribuzione - Non commerciale - Non opere derivate 4.0 Internazionale. 


\title{
MARJOLAINE UNTER ECKER, Questions identitaires dans les récits afropéens de Léonora Miano
}

\author{
Carminella Biondi
}

\section{RÉFÉRENCE}

MARJOLAINE UNTER ECKER, Questions identitaires dans les récits afropéens de Léonora Miano, Toulouse, Presses universitaires du Midi, 2016, 189 pp.

1 Les afropéens sont les habitants d'un nouveau continent frontalier, intérieur, Afropea, né de la rencontre de l'Afrique et de l'Europe, mais «situé à la frontière des lieux, des temps et des êtres» (p. 27). Il s'agit du pays habité par les africains sub-sahariens qui vivent dans le continent européen (dans le cas particulier la France), les afrodescendants, mais aussi de ceux qui vivent ailleurs (même en Afrique), et dont l'histoire, présente et passée, s'est entremêlée à celle de l'Europe. Les antillais en font donc partie. Dans un important recueil de conférences, publié en 2012, Habiter la frontière, Léonora Miano soulignait l'importance de ce lieu de rencontres, qui est presque toujours un lieu cicatriciel, qu'il faut soigner, car la rencontre se fait le plus souvent dans la haine, la violence et le mépris, mais qui est aussi le lieu de naissance d'un sens nouveau, d'un monde nouveau, où les échanges peuvent devenir très féconds (la «frontière habitée» de Léonora Miano n'est pas sans rappeler le «lieu commun» d'Édouard Glissant, car elle aussi est lieu de relation).

Dans son essai, Marjolaine Unter Ecker a choisi de se pencher sur un corpus très bien caractérisé dans la production littéraire de l'écrivaine d'origine camerounaise qui vit depuis les années 1990 à Paris. Elle y étudie cinq romans ou recueils de nouvelles qui sont en large majorité situés à Paris, et dont les protagonistes sont des afrodescendants de la diaspora: Tels des astres éteints (2008), Afropean Soul et autres nouvelles (2008), Blues pour Élise (2010), Ces âmes chagrines (2011), Écrits pour la parole (2013). Il va de soi que tous 
les ouvrages de Miano sont convoqués dans le discours critique, mais la focalisation est bien circonscrite. Ce sont les différentes déclinaisons des modes de vie frontaliers (en particulier des femmes) qui bâtissent la structure romanesque du corpus, et qui font l'objet de l'étude de Marjolaine Unter Ecker. Ces différentes déclinaisons d'histoires individuelles ou collectives, ainsi que l'a bien mis en évidence Marjolaine Unter Ecker, sont autant de questionnements - et parfois de réponses - aux problèmes identitaires que pose la vie dans un espace cicatriciel, un espace existentiel qui n'a plus de références et doit se construire au fur et à mesure. En effet ces identités nouvelles ne se font pas, dans la réalité, à partir d'une quelconque théorie ou d'un modèle, mais à travers une série de tâtonnements qui peuvent amener à la ruine ou au salut (Miano préfère le chemin du salut, mais l'autre est toujours aux aguets et parfois a le dessus). Si les personnages de Miano tâtonnent dans leur difficile quotidien, Marjolaine Unter Ecker, par contre, structure de façon très solide son essai, en se fondant sur un certain nombre de références reconnues, en particulier l'œuvre de Paul Ricœur, qui peuvent parfois donner l'impression de conditionner l'approche critique, mais qui, au contraire, se révèlent être des bases solides sur lesquelles bâtir un parcours d'analyse de textes narratifs visant à conjuguer la beauté esthétique avec la prise en charge des innombrables nuances d'un individuation in fieri, dans des conditions prohibitives.

Dans l'étude de ces identités complexes, la critique privilégie trois aspects, qu'elle analyse dans les trois parties de son essai: l'identité physique, la plus fragile, fondée sur l'apparence (pp.31-74), l'identité intime, qui se penche sur l'intériorité des personnages (pp.75-124), et l'identité historique, collective, qui tient compte des différents apports contribuant à caractériser l'individu dans la société et dans l'histoire (pp.125-170). Il s'agit évidement d'une division artificielle, car toutes ces identités interviennent dans la construction d'un personnage et d'une histoire, et trouvent leur point de raccord dans l'Introduction (pp.9-29) de l'essai et leur synthèse dans la Conclusion (pp.171-178), qui rappelle, en raccourci, l'originalité et la fécondité de la mise en œuvre du questionnement identitaire de la part de Léonora Miano, qui, tout en étant particulier, produit des échos qui nous impliquent tous. Mais elle rappelle aussi la beauté, la force des ouvrages de l'écrivaine, où la problématique fait partie de la construction de l'intrigue et s'y fond, sans l'appesantir.

4 Les problèmes de tout ordre soulevés par les ouvrages de Miano, et bien analysés par Marjolaine Unter Ecker, sont très nombreux, car ils sont au cœur de notre époque: immigration et migrance, stabilité et errance, rencontre de mondes et de cultures, francophonie, importance de l'influence américaine dans la création de modèles, surtout dans le domaine de la musique, etc. Un essai important, que celui de Marjolaine Unter Ecker, qui a le mérite de la clarté, dans son organisation et dans son écriture, et qui, tout en se limitant à un corpus réduit, réussit à nous amener au cœur de l'œuvre d'une grande écrivaine de la diaspora noire, dont les textes plongent leurs racines «dans la douleur du passé, mais en conservant l'espoir du futur» (p. 124). 